Check for updates

Cite this: RSC Adv., 2018, 8, 11367

Received 2nd February 2018

Accepted 16th March 2018

DOI: $10.1039 / c 8 r a 01047 a$

rsc.li/rsc-advances

\title{
Thermal property improvement of polytetrafluoroethylene nanocomposites with graphene nanoplatelets
}

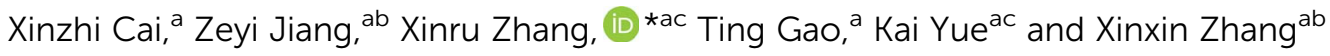

Thermal properties including the crystallization behavior, thermal stability and thermal conductivity for a series of graphene nanoplatelet (GNP)-polytetrafluoroethylene (PTFE) nanocomposites were studied. The GNP-PTFE nanocomposites were fabricated via solvent-assisted blending followed by cold-pressing and sintering. The results indicated that the GNP-PTFE nanocomposites retained the good thermal stability of the PTFE matrix, and possessed better crystallization and much higher thermal conductivity than pure PTFE. The thermal conductivity of PTFE nanocomposites with a GNP mass fraction of $20 \%$ could reach $4.02 \mathrm{~W}\left(\mathrm{~m} \mathrm{~K}^{-1}\right.$, which was increased by $1300 \%$ compared with pure PTFE. Additionally, a theoretical model was proposed to analyze the thermal conductivity of GNP-PTFE nanocomposites. It is demonstrated that adding GNPs into PTFE homogeneously can effectively improve the thermal properties of the nanocomposites.
\end{abstract}

\section{Introduction}

Polytetrafluoroethylene (PTFE) is a kind of important fluoropolymer with a low friction coefficient, high chemical resistance, fire-retardant properties and high thermal stability, which is widely used in various high-end applications, such as aerospace, the automotive industry, chemical engineering, biomedicine and heat management. ${ }^{1-3}$ However, pure PTFE has a low thermal conductivity of $\sim 0.3 \mathrm{~W}(\mathrm{~m} \mathrm{~K})^{-1},{ }^{4}$ which limits its application in engineering. ${ }^{5-7}$ To address this issue, the incorporation of nanofillers with high thermal conductivity into the PTFE matrix has received much attention in the past few years.

To date, researchers have investigated the thermal properties of PTFE composites filled with some nanofillers. Using the finite element method, Jin et al. mathematically analyzed the effects of size, volume fraction, and distribution of fillers on the thermal conductivity of graphite particles-PTFE composites and carbon fibers-PTFE composites. ${ }^{8}$ Chen et al. measured the thermal conductivity of boron nitride (BN)-PTFE, zinc oxide (ZnO)-PTFE, tetra-needle-shaped zinc oxide whiskers (T-ZnO)PTFE and hybrid filler (ZnO and BN, T-ZnO and BN)-PTFE composites. ${ }^{9}$ They found that the hybrid filler-PTFE composites had a higher thermal conductivity than those filled with only one kind of filler at the same content, and the thermal

${ }^{a}$ School of Energy and Environmental Engineering, University of Science and Technology Beijing, Beijing, 100083, China. E-mail: xinruzhang@ustb.edu.cn

${ }^{b}$ Beijing Key Laboratory for Energy Saving and Emission Reduction of Metallurgical Industry, University of Science and Technology Beijing, Beijing, 100083, China

${ }^{`}$ Beijing Engineering Research Center of Energy Saving and Environmental Protection, University of Science and Technology Beijing, Beijing, 100083, China conductivity for the hybrid filler-PTFE composites was $1.38 \mathrm{~W}$ $(\mathrm{m} \mathrm{K})^{-1}$, when the content of T-ZnO and BN was $3 \%$ and $27 \%$, respectively. Recently, Pan et al. reported the thermal conductivity of micro-nano AlN cofilled PTFE composites (mn-AlNPTFE) and silane coupling agent modified BN filled PTFE composites (scam-BN-PTFE). ${ }^{10,11}$ The results indicated that when the volume fraction of filler was $30 \%$, the thermal conductivity of mn-AlN-PTFE and scam-BN-PTFE composites was $0.84 \mathrm{~W}(\mathrm{~m} \mathrm{~K})^{-1}$ and $0.722 \mathrm{~W}\left(\mathrm{~m} \mathrm{~K}^{-1}\right.$, respectively. In addition, Sahli et al. prepared thermally expanded graphitePTFE composites (TEG-PTFE) and studied the thermal stability and thermal resistance of the composites. They found that with the increase of the TEG content, the glass-transition temperature and the degradation temperature of TEG-PTFE both increased. ${ }^{12}$ These researches demonstrated that the incorporating of fillers with high thermal conductivity is effective to improve the thermal properties of PTFE matrix.

Graphene is a kind of two-dimensional layered material with an ultrahigh thermal conductivity of $\sim 5000 \mathrm{~W}(\mathrm{~m} \mathrm{~K})^{-1}$, high Young's modulus of $\sim 1.0 \mathrm{TPa}$ and high intrinsic mobility of $\sim 200000 \mathrm{~cm}^{2}(\mathrm{~V} \mathrm{~s})^{-1},{ }^{13,14}$ which shows a great potential to be a filler for the fabrication of nanocomposites with high properties. ${ }^{15-17} \mathrm{Gu}$ fabricated a series of GNPs-polymer nanocomposites, i.e., GNPs-ultrahigh molecular weight polyethylene, ${ }^{18}$ functionalized GNPs-bisphenol A epoxy resin ${ }^{19}$ and functionalized GNPs-phenylphosphonate based epoxy resin, ${ }^{20}$ and found that GNPs have a strong ability to improve the thermal properties of composites. Recently, some researchers have studied the methods of fabricating graphenePTFE nanocomposite and the effects of graphene filler on the gas barrier behavior, mechanical properties and electrical 
characteristics for the graphene-PTFE nanocomposites. Van Rooyen et al. investigated the method to prepare graphenePTFE nanocomposite and evaluated the influence of the powder blending, fabrication method and the graphene concentration on its physical property. ${ }^{21}$ In addition, van Rooyen et al. studied the gas barrier properties of oxyfluorinated graphene filled PTFE nanocomposites, and found that when the volume fraction of oxyfluorinated graphene was $7 \%$, the helium gas permeability of nanocomposites reduced by $88 \% .{ }^{22}$ Suh and Bae fabricated the graphene-PTFE nanocomposite via high-energy milling of PTFE and graphene powder followed by hotpressing, and measured its mechanical and electrical properties. The results indicated that, compared to pure PTFE, the yield stress and Young's modulus of graphene (3 vol\%)-PTFE nanocomposites increased $60 \%$ and $233 \%$, respectively. ${ }^{23}$ The electrical conductivity of graphene (30 vol\%)-PTFE nanocomposites was $7353 \mathrm{~S} \mathrm{~m}^{-1} \cdot{ }^{24}$ However, to date, little is known about the thermal properties of graphene-PTFE nanocomposites.

In this study, the graphene nanoplatelet (GNP)-PTFE nanocomposites with varied GNP mass fractions of $1 \%, 5 \%, 10 \%$, $15 \%$ and $20 \%$ were fabricated via solvent-assisted blending followed by cold-pressing and sintering. Then, the microstructure, crystallization behavior, thermal stability and thermal conductivity of GNP-PTFE nanocomposites were measured. Finally, based on the Maxwell-Garnett effective medium approach (MGEMA), a mathematical model to analyze and predict the thermal conductivity of GNP-PTFE nanocomposites was developed by incorporating the interface thermal resistance. The findings may have important implications for understanding the thermal properties of GNP-PTFE nanocomposite.

\section{Experimental}

\section{Preparation of GNPs}

The graphene dispersions with different mass fractions were prepared by liquid phase exfoliation (LPE) of graphite sheets (Supplied by Chengdu Organic Chemicals Co. Ltd. China.) in ethanol using a tip sonicator (Scientz-950E, Scientz Biotechnology Co. Ltd, China) under the power of $300 \mathrm{~W}$ for $120 \mathrm{~min} .^{25}$ After that, the morphology, thickness, defects of the produced GNPs were characterized. In detail, the morphology of pristine graphite and the produced GNP was observed by a scanning electron microscopy (SEM) (Nova NanoSEM 430, FEI, USA) operating at a $5 \mathrm{kV}$ acceleration voltage. The thickness of the produced GNP was examined by a transmission electron microscope (TEM) (Tecnai F30, FEI, USA). The defects of the GNPs were measured by Raman spectrometer (LabRAM HR800, Horiba Jobin-Yvon, Japan) with a $514 \mathrm{~nm}$ wavelength laser via depositing a GNP film onto a glass slide.

\section{Fabrication of GNP-PTFE nanocomposites}

The method to fabricate the GNP-PTFE nanocomposites consisted of three steps, as shown in Fig. 1. Firstly, the graphene dispersions with a series of mass fractions were prepared by

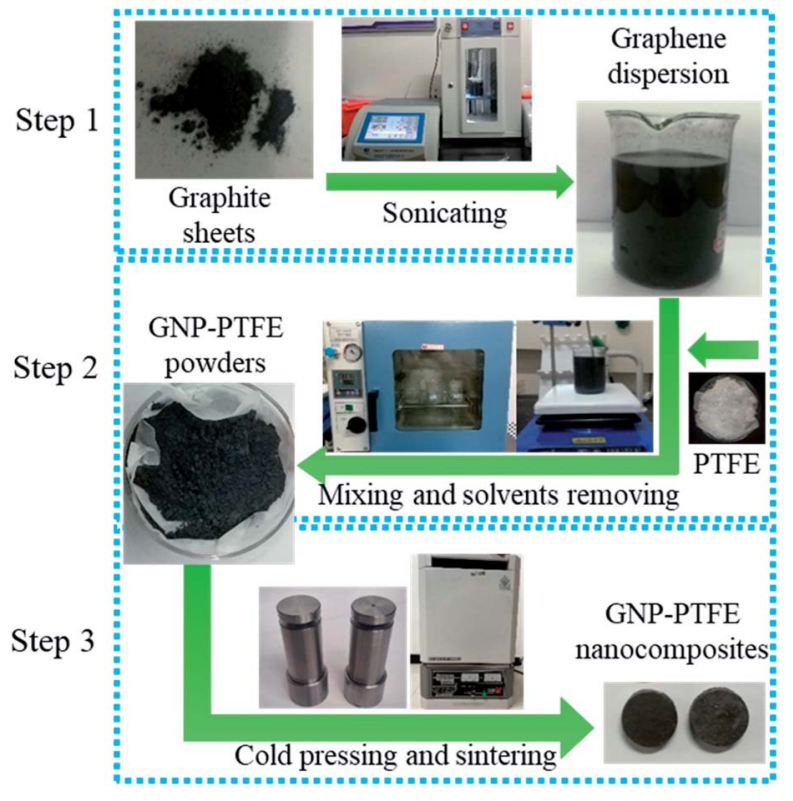

Fig. 1 Schematic of the method to fabricate the GNP-PTFE nanocomposites. Step 1: graphene dispersion was prepared by liquid phase exfoliation of graphite sheets in ethanol via tip sonication. Step 2: PTFE powders were added into the graphene dispersions and then fully mixed with GNPs while removing the ethanol using a heating magnetic stirrer for $6 \mathrm{~h}$ at $60^{\circ} \mathrm{C}$. Subsequently, the slurry was vacuum dried at $60{ }^{\circ} \mathrm{C}$ for $24 \mathrm{~h}$ to remove the residual ethanol in the GNP-PTFE powder. Step 3: the GNP-PTFE nanocomposites were fabricated by cold pressing with a mould and sintering.

exfoliating graphite sheets in ethanol via tip sonicating. Secondly, the PTFE powders (Obtained from DuPont, USA.) were added into the graphene dispersions and then fully mixed with GNPs while removing the ethanol using a heating magnetic stirrer for $6 \mathrm{~h}$ at $60{ }^{\circ} \mathrm{C}$. After that, the GNP-PTFE slurry was vacuum dried at $60{ }^{\circ} \mathrm{C}$ for $24 \mathrm{~h}$ to remove the residual ethanol. Finally, the GNP-PTFE powders were cold pressed into cylindrical samples with a diameter of $15 \mathrm{~mm}$ at the pressure of $40 \mathrm{MPa}$ for about $10 \mathrm{~min}$. And then the performed GNP-PTFE samples were put into a muffle oven for sintering. During the sintering, the temperature in the oven was firstly increased to $380{ }^{\circ} \mathrm{C}$ in $5 \mathrm{~h}$, and held at $380^{\circ} \mathrm{C}$ for $3 \mathrm{~h}$, then decreased slowly to room temperature. In the experiments, the PTFE nanocomposites with the GNP mass fractions of $1 \%, 5 \%, 10 \%, 15 \%$ and $20 \%$ were fabricated.

\section{Characterization of GNP-PTFE nanocomposites}

The microstructure, crystallization behavior, thermal stability, thermal conductivity and heat transfer phenomena of GNPPTFE nanocomposites were characterized. In detail, the microstructure of GNP-PTFE nanocomposites and the homogeneity of GNPs in PTFE matrix were micro-observed by metallographic microscope (Axio Scope A1, Zeiss, Germany) and SEM. It should be noted that, in order to observe the microstructure precisely, the GNP-PTFE nanocomposites were fractured in liquid nitrogen to form a neat cross section. The crystallization behavior and thermal stability of GNP-PTFE nanocomposites were determined with different scanning 
calorimeter (DSC) and thermogravimetric analysis (TGA) by a thermal analysis platform (Labsys Evo, Setaram, France) at a heating rate of $10{ }^{\circ} \mathrm{C} \mathrm{min}^{-1}$ from $25{ }^{\circ} \mathrm{C}$ to $900{ }^{\circ} \mathrm{C}$ under a nitrogen atmosphere. In addition, the thermal conductivity of GNP-PTFE nanocomposites was measured by the transient plane heat source method using a commercial thermal conductivity meter (2500 s, Hot disc, Sweden). In detail, the measurements were conducted by putting the sensor (diameter: $3 \mathrm{~mm}$ ) into two similar cylindrical samples (diameter: $15 \mathrm{~mm}$, height: $6 \mathrm{~mm}$ ). A heat pulse of $0.02 \mathrm{~W}$ for 20 seconds was supplied by the sensor. The temperature changes of the samples were recorded and then the thermal conductivity was determined. ${ }^{26}$ During the measurements, six samples were used. Each measurement was repeated for at least 3 times, and results were shown in mean \pm standard deviation. Moreover, to observe the heat transfer phenomena through the GNP-PTFE nanocomposites, the pure PTFE sample and GNP-PTFE nanocomposites samples were placed on a heating plate with a constant temperature of $150{ }^{\circ} \mathrm{C}$. The temperature distribution of the samples was measured with an infrared thermal imaging instrument (E60, FLIR Systems, USA).

\section{Predicting the thermal conductivity of GNP-PTFE nanocomposites with a mathematical model}

A modified Maxwell-Garnett effective medium approach (MGEMA) is used to predict the thermal conductivity of GNPPTFE nanocomposites by considering the size and aspect ratio of GNP, the thermal conductivity of GNP in each direction and the interface thermal resistance between the GNPs and PTFE matrix. ${ }^{27,28}$ Specifically, as shown in Fig. 2, the GNP in nanocomposites was treated as a flat cylinder with a diameter of $a_{1}$ (or $a_{2}, a_{1}=a_{2}$ ) and a height of $a_{3}$, where 1 and 2 represented in-plane direction, 3 represented throughplane direction. Considering the interfacial thermal resistance between the GNP and polymer matrix, the GNP and the interfacial thermal barrier were taken as a composite unit cell. The thermal conductivity of the unit $K_{i}^{\mathrm{c}}$ can be expressed as,

$$
K_{i}^{\mathrm{c}}=\frac{K_{i}}{1+\frac{a_{\mathrm{k}} K_{i}}{a_{i} K_{m}}}
$$

where, $K_{i}$ is the thermal conductivity of GNP along the $a_{i}(i=1$, $2,3)$ direction, $K_{m}$ is the thermal conductivity of matrix, $a_{\mathrm{k}}$ is known as Kapitza radius, which defined by,

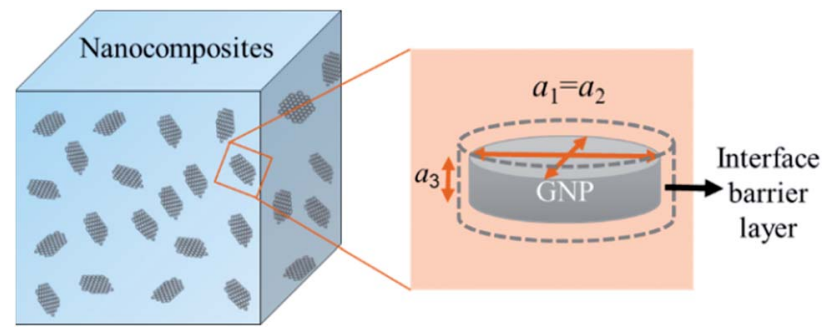

Fig. 2 Schematic for a composite unit of a GNP coated with a thin interfacial thermal barrier layer.

$$
a_{\mathrm{k}}=R_{\mathrm{k}} K_{m}
$$

where, $R_{\mathrm{k}}$ is the interface thermal resistance between the GNP and polymer matrix. Orientation distribution of the GNP in nanocomposites was assumed randomly. The thermal conductivity of nanocomposites, $K^{*}$, can be expressed as,

$$
K^{*}=K_{m} \frac{3+f\left[2 \beta_{1}\left(1-L_{1}\right)+\beta_{3}\left(1-L_{3}\right)\right]}{3-f\left[2 \beta_{1} L_{1}+\beta_{3} L_{3}\right]}
$$

with

$$
\beta_{i}=\frac{K_{i}^{\mathrm{c}}-K_{m}}{K_{m}+L_{i}\left(K_{i}^{\mathrm{c}}-K_{m}\right)}
$$

where $f$ is the volume fractions of GNP in nanocomposites; $L_{i}(i=1,2,3)$ is the geometrical factor, which is dependent on the aspect ratio of GNP, and can be expressed as,

$$
L_{1}=L_{2}=\frac{\pi\left(a_{3} / a_{1}\right)}{4} \quad L_{3}=1-2 L_{1}
$$

\section{Results and discussion}

\section{Properties of the produced GNP}

The morphology, thickness, defects of the produced GNPs, were determined by different methods. Fig. 3(a) shows the SEM image of the pristine graphite, which indicates that the graphite sheets are thick and have a size of about $20 \mu \mathrm{m}$. Fig. 3(b) is the SEM image of the produced GNP by exfoliating graphite sheets in ethanol via tip sonicating. It can be found that the produced GNP has a size of about $1.5 \mu \mathrm{m}$. Fig. 3(c) is the TEM image for the produced GNP, which indicates that the GNPs are fewlayered. Fig. 3(d) is the electron diffraction pattern of the produced GNP under the incidence angles of $0^{\circ}$, which provides a six-fold symmetry pattern and shows a typical crystal structure of GNP. ${ }^{29}$ Evidently, Fig. 3(c) and (d) indicate that, the pristine graphite sheets have been exfoliated into few-layer GNPs.

Furthermore, the defects of the produced GNPs were determined by the Raman spectroscopy. Generally, the ratio of intensity of the $\mathrm{D}$ band $\left(1350 \mathrm{~cm}^{-1}\right)$ to $\mathrm{G}$ band $\left(1580 \mathrm{~cm}^{-1}\right)$ is used to characterize the defects of GNP. ${ }^{30}$ The less of $I(\mathrm{D}) / I(\mathrm{G})$, the lower defect density of GNPs. Fig. 3(e) shows a typical Raman spectroscopy of the pristine graphite sheets and the GNP produced by the exfoliation of graphite via tip sonicating. It can be found that the $I(\mathrm{D}) / I(\mathrm{G})$ of the pristine graphite sheets is 0.12 , while the $I(\mathrm{D}) / I(\mathrm{G})$ of the produced GNP is 0.17 . Evidently, the $I(\mathrm{D}) / I(\mathrm{G})$ of the produced GNPs is close to that of the pristine graphite sheets, which indicates that comparing with the pristine graphite, the defects of produced GNPs increase little and the GNPs have high quality.

\section{Microstructure of GNP-PTFE composites}

As shown in Fig. 4, the microstructure of GNP-PTFE nanocomposites was micro-observed by metallographic microscope and SEM. Fig. 4(a)-(c) show the metallographic microscope images for the surface of pure PTFE, GNP-PTFE nanocomposites with 10\% GNP and 20\% GNP, respectively. 

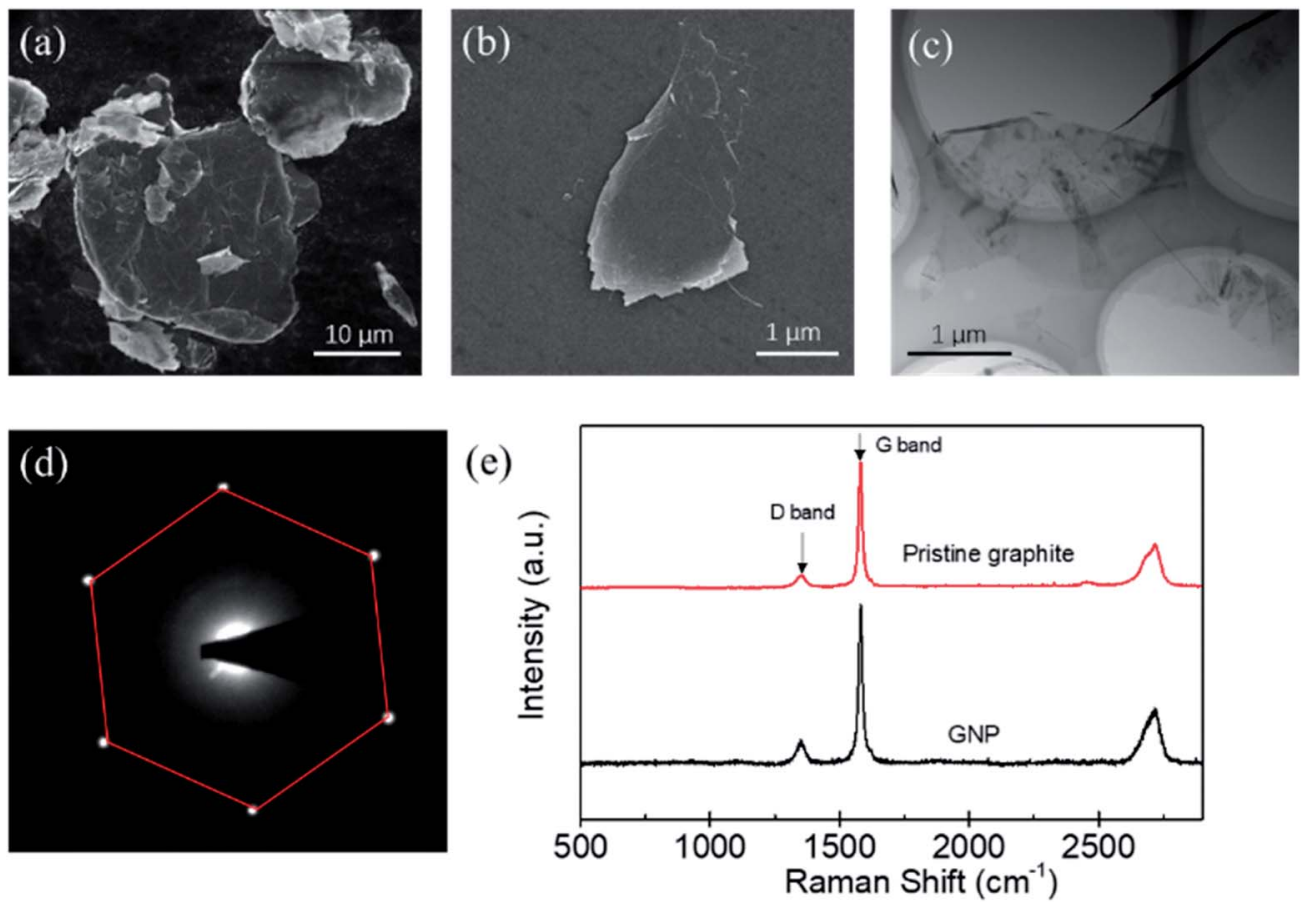

Fig. 3 The SEM image of the pristine graphite (a) and the produced GNP (b). TEM image (c) and the electron diffraction pattern (d) of the produced GNP. (e) Typical Raman spectra for the pristine graphite and the produced GNP by liquid phase exfoliation via tip sonicating.

Fig. 4(d)-(f) show the fractured surface morphologies for the pure PTFE, GNP-PTFE nanocomposites with 10\% GNP and $20 \%$ GNP, respectively. In detail, the Fig. 4(a) and (d) indicate that the pure PTFE has a smooth surface without any impurities. Furthermore, the homogeneity of GNPs in PTFE matrix could be observed in Fig. 4(b) and (c), in which the bright and the dark blocks present the GNPs and PTFE matrix in nanocomposites, respectively. Moreover, the Fig. 4(b) and (e) indicate that, for the GNP (10\%)-PTFE nanocomposites, the GNP fillers disperse homogeneously, and are isolated with each other. Meanwhile, when the mass fractions of GNP increased to $20 \%$, as shown in the Fig. 4(c) and (f), more GNPs could be

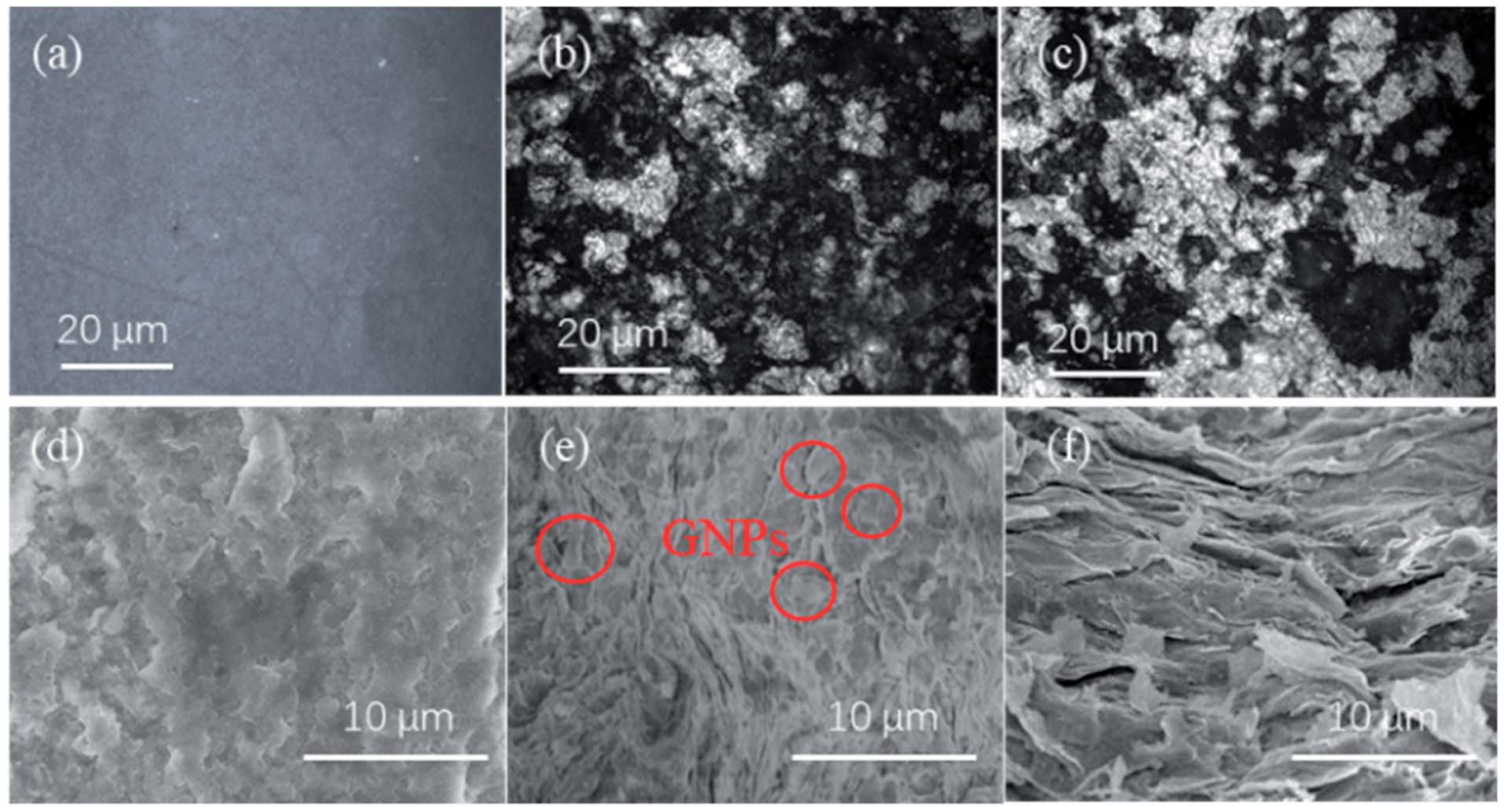

Fig. 4 Metallographic microscope images for the surface of pure PTFE (a), 10\% GNP-PTFE nanocomposite (b) and 20\% GNP-PTFE nanocomposite (c). SEM images for the surface of pure PTFE (d), 10\% GNP-PTFE nanocomposite (e) and 20\% GNP-PTFE nanocomposite (f). 
observed in the same range of view, meanwhile, some GNPs are found to contact with each other.

\section{Crystalline behavior and thermal stability}

PTFE is a kind of semicrystalline polymer with crystalline structure. Generally, the crystalline structure of the polymer matrix is an important factor influencing the thermal properties of nanocomposites. ${ }^{31}$ In the experiment, to quantitatively analyze the crystalline behavior, the degree of crystallinity $\left(X_{\mathrm{c}}\right)$ for the pure PTFE and GNP-PTFE nanocomposites is calculated by,

$$
X_{\mathrm{c}}=\frac{\Delta H_{\mathrm{m}}}{\Delta H_{\mathrm{m}}^{\infty}(1-\alpha)}
$$

where $\Delta H_{\mathrm{m}}$ is the measured fusion heat, $\Delta H_{\mathrm{m}}^{\infty}$ is the fusion heat of $100 \%$ crystalline PTFE, which is equal to $69 \mathrm{~J} \mathrm{~g}^{-1,32} \alpha$ is the mass fraction of GNP.

Fig. 5(a), (b) and (c) show the DSC curves, the melting temperature $\left(T_{\mathrm{m}}\right)$ and the degree of crystallinity $\left(X_{\mathrm{c}}\right)$ for the pure PTFE and GNP-PTFE nanocomposites. It can be found that $T_{\mathrm{m}}$ of all PTFE nanocomposites ranged from $317.1{ }^{\circ} \mathrm{C}$ to $317.9{ }^{\circ} \mathrm{C}$, which approximately equal to that of pure PTFE $\left(\sim 317.8{ }^{\circ} \mathrm{C}\right)$. The results indicate that the presence of GNP induces little change for the $T_{\mathrm{m}}$ of PTFE nanocomposites. Moreover, Fig. 5(c) shows that the $X_{\mathrm{c}}$ of GNP-PTFE nanocomposites are higher than that of the pure PTFE, which indicates that the GNP can improve the crystallization behavior of PTFE nanocomposites. In detail, when the mass fractions of GNP increase from $0 \%$ to $15 \%$, the $X_{\mathrm{c}}$ for the pure PTFE and GNP-PTFE nanocomposites increase from $31.65 \%$ to $41.69 \%$. The results indicate that GNP has a nucleating effect for the PTFE nanocomposites during the process of crystallization. However, it should be noted that when the mass fraction of GNP increases to $20 \%$, the $X_{\mathrm{c}}$ decreases to $40.52 \%$. It is likely that the increase of GNPs obstructs the mobility of the PTFE chains. ${ }^{33}$

The thermal stability of nanocomposites is an important parameter in various applications. Fig. 6(a) shows the TGA curves of PTFE nanocomposites, which could be used to evaluate the thermal stability. It can be found that the pure PTFE and the five GNP-PTFE nanocomposites all show a typical onestep decomposition behavior between $500{ }^{\circ} \mathrm{C}$ to $615{ }^{\circ} \mathrm{C}$, which indicates that the increase of GNPs can not influence the behavior of thermal decomposition of PTFE matrix. In addition, to analyse the thermal ability quantitatively, the heat-resistance index (THRI) is calculated. ${ }^{34,35}$ As shown in Table 1 , it can be found that the THRI decreases with the increase of GNPs mass fractions. Specifically, THRI for pure PTFE, 10\% GNPs-PTFE and $20 \%$ GNPs-PTFE nanocomposites are $272.5{ }^{\circ} \mathrm{C}, 269.8{ }^{\circ} \mathrm{C}$ and $269.4{ }^{\circ} \mathrm{C}$, respectively. This indicates that the initial decomposition temperature of PTFE nanocomposites decreases with the addition of GNPs into PTFE matrix. This may be due to two reasons. On one hand, since the thermal conductivity of GNPs is much higher than that of polymer matrix, the enhanced thermal conduction in the presence of GNPs may accelerate the decomposition of matrix, which can reduce the decomposition temperatures. ${ }^{36,37}$ On the other hand, the difference of surface
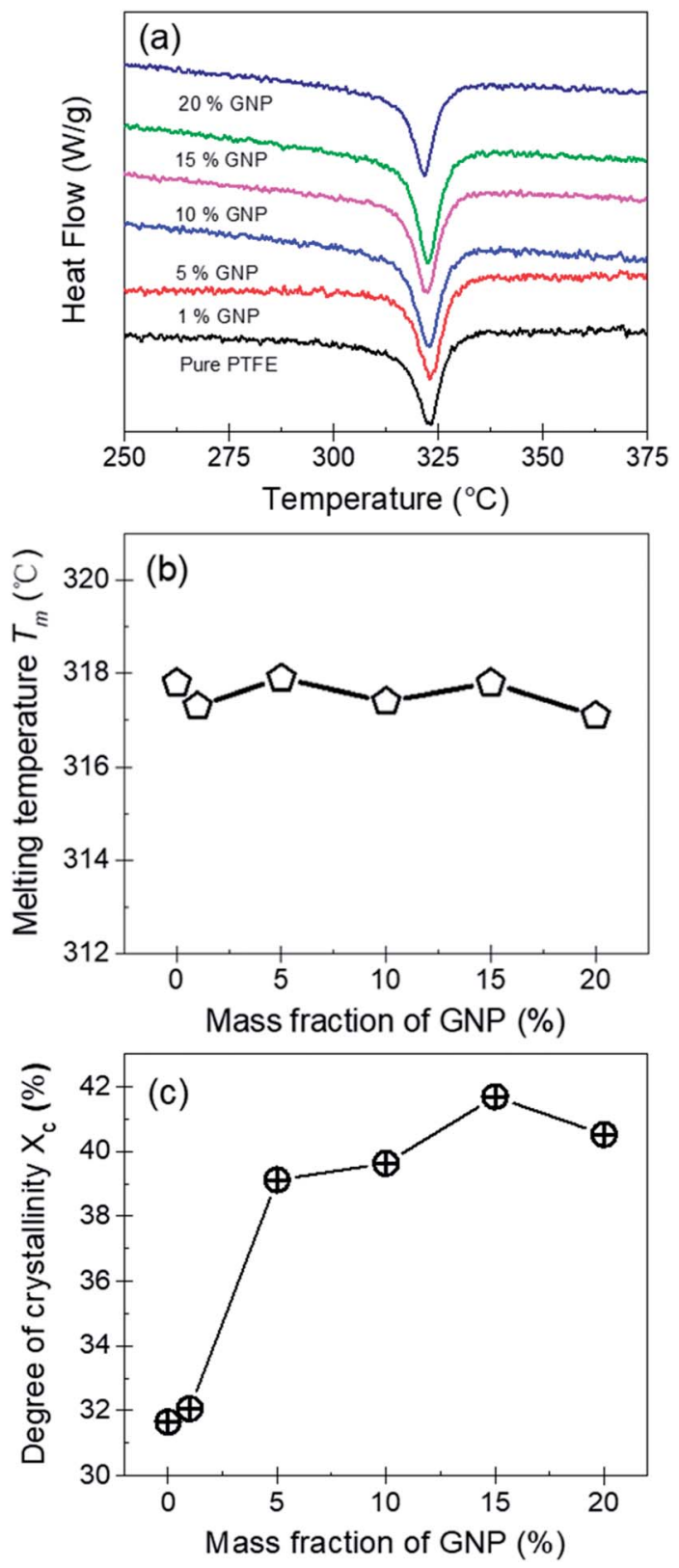

Fig. 5 (a) DSC curves of pure PTFE and GNP-PTFE nanocomposites. Melting temperature $T_{m}(b)$ and the degree of crystallinity $X_{c}$ (c) for pure PTFE and GNP-PTFE nanocomposites as a function of mass fraction of GNP.

characteristics between polymer matrix and fillers may induce weak interfacial interactions between them. ${ }^{\mathbf{3 8 , 3 9}}$ Thus, there may be some defects in the composites, which also can decrease the decomposition temperatures of composites. Moreover, Fig. 6(b) shows the residual mass fraction of PTFE nanocomposites as a function of the mass fractions of GNP. Generally, GNPs could be stable at $800{ }^{\circ} \mathrm{C}$ in nitrogen. Therefore, the actual mass fraction of GNP can be estimated with the residual mass fraction. It can be found that the actual residual mass fraction of GNP agrees well with the initial designed mass fractions of GNP, which also implies that the GNPs disperse uniformly in PTFE matrix. 

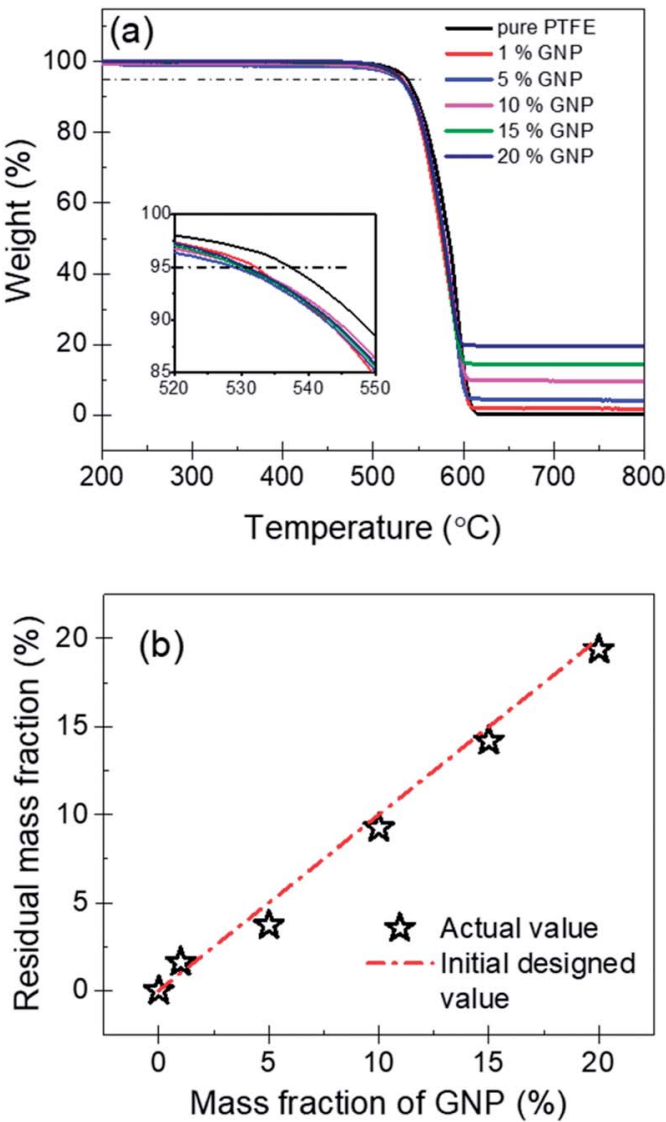

Fig. 6 (a) TGA curves of pure PTFE and GNP-PTFE nanocomposites. (b) Actual residual mass fraction of PTFE nanocomposites as a function of the initial designed mass fractions of GNP.

Table 1 Thermal data of the pure PTFE matrix and the GNPs-PTFE nanocomposites from TGA analysis

\begin{tabular}{llll}
\hline & \multicolumn{2}{l}{$\begin{array}{l}\text { Weight loss } \\
\text { temperature } /{ }^{\circ} \mathrm{C}\end{array}$} & \\
\cline { 2 - 3 } Samples & $T_{5}$ & $T_{30}$ & $\begin{array}{l}\text { The heat resistance } \\
\text { index (THRI) }{ }^{a} /{ }^{\circ} \mathrm{C}\end{array}$ \\
\hline Pure PTFE & 537.3 & 568.8 & 272.5 \\
1\% GNPs & 532.1 & 562.6 & 269.7 \\
5\% GNPs & 529.1 & 565.4 & 269.9 \\
10\% GNPs & 530.4 & 564.1 & 269.8 \\
15\% GNPs & 530.1 & 563.9 & 269.7 \\
$20 \%$ GNPs & 529.7 & 563.2 & 269.4
\end{tabular}

${ }^{a}$ The heat resistance index $(\mathrm{THRI})=0.49\left[T_{5}+0.6\left(T_{30}-T_{5}\right)\right], T_{5}, T_{30}$ is the decomposing temperature at $5 \%, 30 \%$ weight loss, respectively. ${ }^{34}$

\section{Thermal conductivity and heat transfer phenomenon}

Fig. 7(a) shows the thermal conductivity of the PTFE nanocomposites with the GNP mass fractions of $0 \%, 1 \%, 5 \%, 10 \%$, $15 \%$ and $20 \%$. It indicates that the pure PTFE shows a low thermal conductivity of $0.32 \mathrm{~W}(\mathrm{~m} \mathrm{~K})^{-1}$. With the increase of the mass fraction of GNP, the thermal conductivity of GNP-PTFE nanocomposites increases significantly. When the GNP mass fraction is $20 \%$, the effective thermal conductivity of GNP-PTFE nanocomposites reaches $4.02 \mathrm{~W}(\mathrm{~m} \mathrm{~K})^{-1}$, which presents a significant increase of $1300 \%$ compared with that of pure PTFE matrix. Additionally, it can be found that the thermal conductivity of GNP-PTFE nanocomposites is much higher than that of BN-PTFE, AlN-PTFE, ZnO-PTFE nanocomposites, when the fillers have the same content. The results indicate that the incorporating of GNP is effective to improve the thermal conductivity of PTFE matrix, and these GNP-PTFE nanocomposites may have important applications in heat management.

Furthermore, it should be noted that, the thermal conductivity of GNP-PTFE nanocomposites does not increase linearly with the mass fraction of GNP. Especially, when the mass fraction of GNP reaches $20 \%$, the thermal conductivity of GNPPTFE nanocomposites increases higher than that of nanocomposites with the mass fraction of GNP lower than $20 \%$. It is likely that, when the mass fraction of GNP is $20 \%$, as shown in the Fig. 4(c) and (f), some GNPs contact with each other, thus,
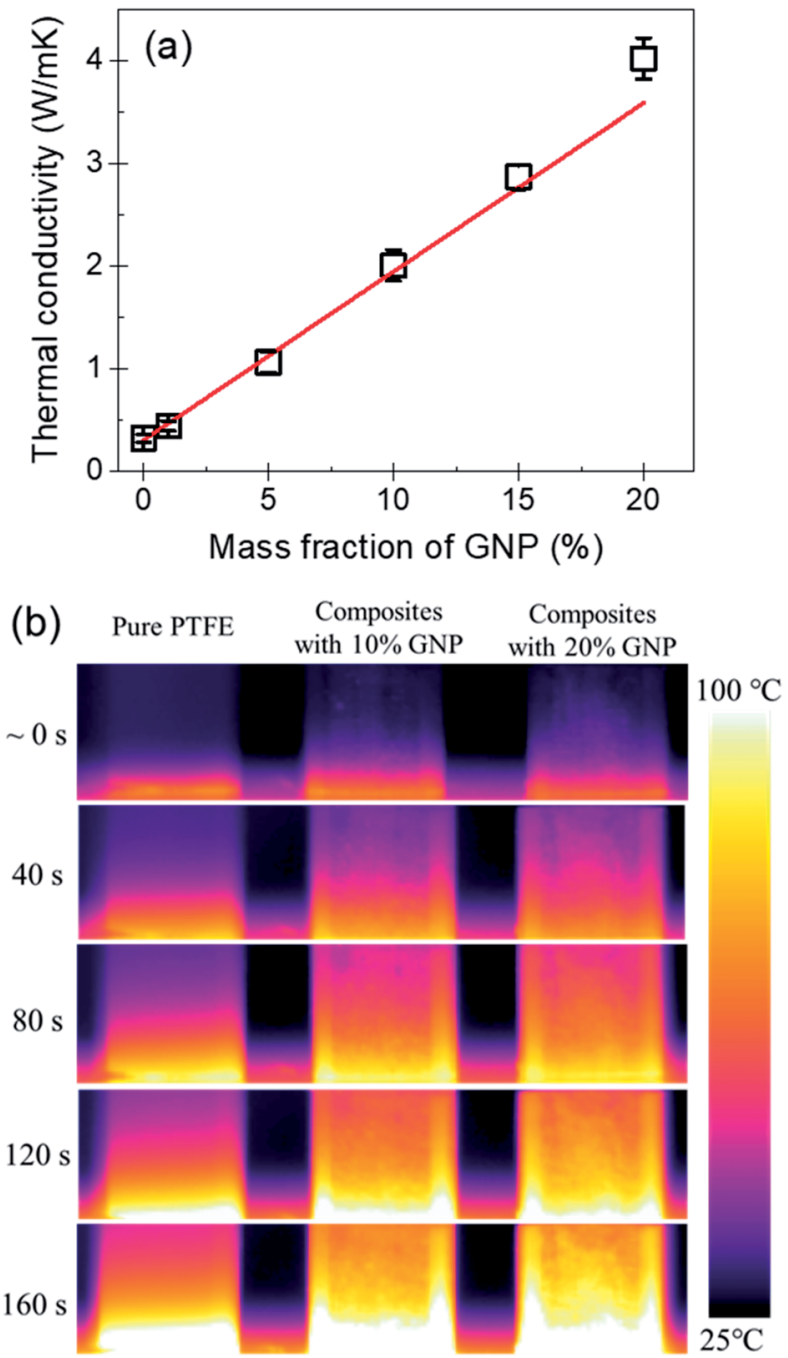

Fig. 7 (a) Thermal conductivity of PTFE nanocomposites as a function of mass fraction of GNP. (b) Temperature distributions for pure PTFE and PTFE nanocomposites with the GNP mass fractions of $10 \%$ and $20 \%$. 
a thermal conductive path may be formed. Unambiguously, these thermal conductive paths contributes to increase the thermal conductivity of GNP-PTFE nanocomposites.

Fig. 7(b) shows the temperature distributions measured with an infrared thermal imaging instrument as a function of heating time for the pure PTFE, GNP-PTFE nanocomposites with GNP content of $10 \%$ and $20 \%$. It is clear that the temperatures at the top of the two GNP-PTFE nanocomposites were much higher than that of the pure PTFE. This indicates that, the heat transfer rate of the PTFE nanocomposites with a GNP content of $20 \%$ is highest, followed by the PTFE nanocomposites with a GNP content of $10 \%$ and the pure PTFE. The results vividly demonstrate that the addition of GNPs into the PTFE can improve the thermal conductivity of matrix and thus enhance the heat transfer through the nanocomposites.

\section{Theoretical analysis for the thermal conductivity of GNP- PTFE nanocomposites with a modified MGEMA}

Based on the SEM and TEM observation shown in Fig. 3(b) and (c), the produced GNPs by exfoliation of graphite via tip sonication has a size of $\sim 1.5 \mu \mathrm{m}$ and shows few-layered. Therefore, in the MGEMA model, the value of $a_{1}$ and $a_{2}$ are set to $1.5 \mu \mathrm{m}$ and $a_{3}$ is assumed as $10 \mathrm{~nm}$. It should be noted that, in this study, we characterized the thickness of GNPs by determining the thickness of the folded edges of GNPs based on about 50 SEM images. The results indicate that the average thickness of GNPs produced by exfoliating is about $10 \mathrm{~nm}$. Furthermore, according to our thermal conductivity measurements for pure PTFE, $K_{m}$ is set to $0.32 \mathrm{~W}(\mathrm{~m} \mathrm{~K})^{-1}$. Based on Yu's results about the thermal conductivities of GNP in-plane and through-plane directions, $K_{1}$ and $K_{2}$ are set to $3000 \mathrm{~W}(\mathrm{~m} \mathrm{~K})^{-1}$ and $K_{3}$ is set to $6 \mathrm{~W}(\mathrm{~m} \mathrm{~K})^{-1} \cdot{ }^{40} \mathrm{In}$ addition, according to Nan's results about the thermal interfacial resistance between the fillers and matrix, ${ }^{41} R_{\mathrm{k}}$ is set ranging from $4 \times 10^{-8}\left(\mathrm{~m}^{2} \mathrm{~K}\right) \mathrm{W}^{-1}$ to $9 \times 10^{-8}$ $\left(\mathrm{m}^{2} \mathrm{~K}\right) \mathrm{W}^{-1}$. Moreover, it should be noted that, to predict the thermal conductivity of nanocomposites using MGEMA, the mass fractions of GNP $(\alpha)$ need to be transformed to the volume fractions $(f)$ by the followed expression,

$$
f=\frac{\rho_{\mathrm{p}}}{\rho_{\mathrm{p}}+\rho_{\mathrm{g}}\left(\frac{1}{\alpha}-1\right)}
$$

where, $\rho_{\mathrm{p}}$ and $\rho_{\mathrm{g}}$ is the density of PTFE and graphene, respectively. Based on the properties of the materials, $\rho_{\mathrm{p}}$ and $\rho_{\mathrm{g}}$ are set to $2.26 \mathrm{~g} \mathrm{~cm}^{-3}$ and $1.59 \mathrm{~g} \mathrm{~cm}^{-3}$, respectively. Based on the eqn (7), the mass fractions of $1 \%, 5 \%, 10 \%, 15 \%$ and $20 \%$ were transformed to the volume fractions of $1.42 \%, 6.98 \%, 13.67 \%, 20.09 \%$ and $26.27 \%$, respectively.

Fig. 8 is the comparison between the thermal conductivity measured in experimental and predicted value by MGEMA. As shown in Fig. 8, it can be found that $R_{\mathrm{k}}$ is a key parameter in the mathematical model, and the thermal conductivity of GNPPTFE nanocomposites decreased with the increase of $R_{\mathrm{k}}$. When the $R_{\mathrm{k}}$ equals to $5.5 \times 10^{-8}\left(\mathrm{~m}^{2} \mathrm{~K}\right) \mathrm{W}^{-1}$, the thermal conductivity of nanocomposites predicted by the modified MGEMA is quite correlated with the experimental results. However, it should be

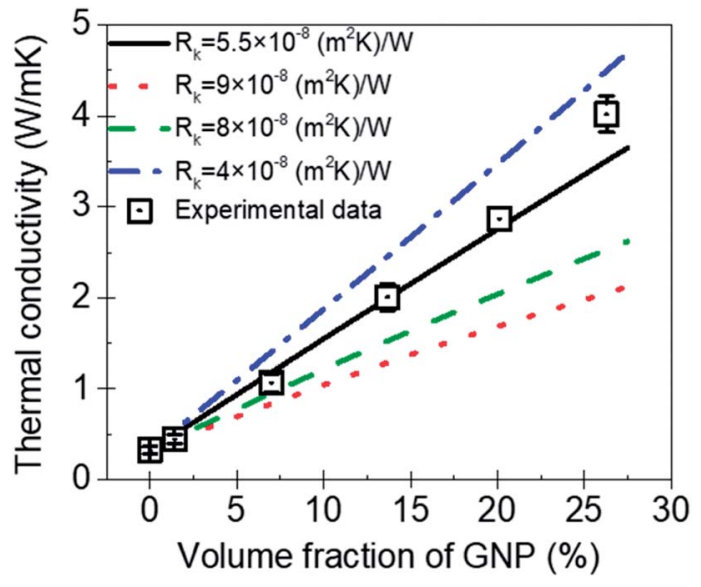

Fig. 8 The thermal conductivity of GNP-PTFE nanocomposites predicted by a modified MGEMA.

noted that, when the volume fraction of GNP reaches to $20 \%$, there is a certain difference between the thermal conductivity of the experimental data and that of the predicted values. This may be due to the fact that when the graphene content is high, the contacts of GNP fillers are not considered in the mathematical model. Nevertheless, the results indicate that the thermal conductivity of nanocomposites can be effectively predicted by the modified MGEMA when the volume fractions of GNP are below $20 \%$.

\section{Conclusions}

The PTFE nanocomposites with the GNP mass fractions of $1 \%$, $5 \%, 10 \%, 15 \%$ and $20 \%$ were fabricated via solvent-assisted blending, cold-pressing and sintering. The thermal properties of these nanocomposites, including crystallization behavior, thermal stability and thermal conductivity were studied. The results indicate that the GNP fillers disperse homogeneously in the PTFE matrix. The melting temperature of all PTFE nanocomposites ranges from $317.1{ }^{\circ} \mathrm{C}$ to $317.9{ }^{\circ} \mathrm{C}$, which approximately equals to that of pure PTFE $\left(\sim 317.8^{\circ} \mathrm{C}\right)$. The degree of crystallinity for GNP-PTFE nanocomposites is higher than that of pure PTFE, indicating that the GNPs improve the crystallization behavior of PTFE nanocomposites. Moreover, the GNPPTFE nanocomposites show a typical one-step decomposition behavior and degrade between $500{ }^{\circ} \mathrm{C}$ to $615{ }^{\circ} \mathrm{C}$. Furthermore, the thermal conductivity of PTFE nanocomposites increases with increasing GNP content. When the mass fraction of GNP is $20 \%$, the thermal conductivity of PTFE nanocomposites reaches $4.02 \mathrm{~W}(\mathrm{~m} \mathrm{~K})^{-1}$, which increase by $1300 \%$ comparing with the pure PTFE. A modified MGEMA model can analyze and predict the thermal conductivity of GNP-PTFE nanocomposites successfully. The results demonstrated that the addition of GNPs into PTFE can not only maintain the good thermal stability of the PTFE, but also improve the degree of crystallinity and thermal conductivity of nanocomposites significantly. The findings may have important implications to understand the 
thermal property of GNP-PTFE nanocomposite toward its application in heat management.

\section{Conflicts of interest}

There are no conflicts to declare.

\section{Acknowledgements}

This work was supported by the National Key Research and Development Program of China (No. 2016YFB0600704), and the Fundamental Research Funds for the Central Universities (No. FRF-TP-16-062A1).

\section{Notes and references}

1 S. Ebnesajjad, Fluoroplastics, Volume 1: Non-Melt Processible Fluoropolymers-The Definitive User's Guide and Data Book, Elsevier, 2014.

2 A. Sarani, N. De Geyter, A. Y. Nikiforov, R. Morent, C. Leys, J. Hubert and F. Reniers, Surf. Coat. Technol., 2012, 206, 2226-2232.

3 J. Zhang, X. Yu, H. Li and X. Liu, Appl. Surf. Sci., 2002, 185, 255-261.

4 D. M. Price and M. Jarratt, Thermochim. Acta, 2002, 392, 231236.

5 A. L. Moore and L. Shi, Mater. Today, 2014, 17, 163-174.

6 H. Chen, V. V. Ginzburg, J. Yang, Y. Yang, W. Liu, Y. Huang, L. Du and B. Chen, Prog. Polym. Sci., 2016, 59, 41-85.

7 P. Zhang, J. Zeng, S. Zhai, Y. Xian, D. Yang and Q. Li, Macromol. Mater. Eng., 2017, 302, 1700068.

8 Z. Jin, X. Chen, Y. Wang and D. Wang, Comput. Mater. Sci., 2015, 102, 45-50.

9 B. Chen, D. Luan, J. Huang and J. Zhang, J. Appl. Polym. Sci., 2015, 132, 42302.

10 C. Pan, K. Kou, Q. Jia, Y. Zhang, Y. Wang, G. Wu and A. Feng, J. Mater. Sci.: Mater. Electron., 2016, 27, 11909-11916.

11 C. Pan, K. Kou, Q. Jia, Y. Zhang, G. Wu and T. Ji, Composites, Part B, 2017, 111, 83-90.

12 M. Sahli, A. Cablé, K. Chetehouna, S. Hamamda, N. Gascoin and S. Revo, Composites, Part B, 2017, 124, 175-181.

13 A. K. Geim and K. S. Novoselov, Nat. Mater., 2007, 6, 183-191.

14 Y. Zhu, S. Murali, W. Cai, X. Li, J. W. Suk, J. R. Potts and R. S. Ruoff, Adv. Mater, 2010, 22, 3906-3924.

15 K. M. Shahil and A. A. Balandin, Nano Lett., 2012, 12, 861867.

16 X. Zhang, B. R. S. Rajaraman, H. Liu and S. Ramakrishna, RSC Adv., 2014, 4, 28987-29011.

17 W. K. Chee, H. N. Lim, N. M. Huang and I. Harrison, RSC Adv., 2015, 5, 68014-68051.
18 J. Gu, N. Li, L. Tian, Z. Lv and Q. Zhang, RSC Adv., 2015, 5, 36334-36339.

19 J. Gu, X. Yang, Z. Lv, N. Li, C. Liang and Q. Zhang, Int. J. Heat Mass Transfer, 2016, 92, 15-22.

20 J. Gu, C. Liang, X. Zhao, B. Gan, H. Qiu, Y. Guo, X. Yang, Q. Zhang and D.-Y. Wang, Compos. Sci. Technol., 2017, 139, 83-89.

21 L. J. van Rooyen, H. Bissett, M. C. Khoathane and J. KargerKocsis, J. Appl. Polym. Sci., 2016, 133, 43369.

22 L. J. van Rooyen, H. Bissett, M. C. Khoathane and J. KargerKocsis, Carbon, 2016, 109, 30-39.

23 J. Suh and D. Bae, Composites, Part B, 2016, 95, 317-323.

24 J. Y. Suh, S. E. Shin and D. H. Bae, J. Compos. Mater., 2016, 51, 2565-2573.

25 Y. Arao and M. Kubouchi, Carbon, 2015, 95, 802-808.

26 X. Yang, L. Tang, Y. Guo, C. Liang, Q. Zhang, K. Kou and J. Gu, Composites, Part A, 2017, 101, 237-242.

27 C.-W. Nan, R. Birringer, D. R. Clarke and H. Gleiter, J. Appl. Phys., 1997, 81, 6692-6699.

28 C. W. Nan, Z. Shi and Y. Lin, Chem. Phys. Lett., 2003, 375, 666-669.

29 I. W. P. Chen, Y.-S. Chen, N.-J. Kao, C.-W. Wu, Y.-W. Zhang and H.-T. Li, Carbon, 2015, 90, 16-24.

30 H. C. Lee, W.-W. Liu, S.-P. Chai, A. R. Mohamed, A. Aziz, C.-S. Khe, N. M. S. Hidayah and U. Hashim, RSC Adv., 2017, 7, 15644-15693.

31 C. T'Joen, Y. Park, Q. Wang, A. Sommers, X. Han and A. Jacobi, Int. J. Refrig., 2009, 32, 763-779.

32 Z. Dong-na, K. Kai-chang, Z. Yu, Z. Qing-xin and Z. Zhenchao, J. Appl. Polym. Sci., 2013, 130, 3710-3717.

33 C. Pan, K. Kou, G. Wu, Y. Zhang and Y. Wang, J. Mater. Sci.: Mater. Electron., 2015, 27, 286-292.

34 C. Liang, P. Song, H. Gu, C. Ma, Y. Guo, H. Zhang, X. Xu, Q. Zhang and J. Gu, Composites, Part A, 2017, 102, 126-136.

35 J. Gu, W. Dong, Y. Tang, Y. Guo, L. Tang, J. Kong, S. Tadakamalla, B. Wang and Z. Guo, J. Mater. Chem. C, 2017, 5, 6929-6936.

36 Y. R. Lee, A. V. Raghu, H. M. Jeong and B. K. Kim, Macromol. Chem. Phys., 2009, 210, 1247-1254.

37 J. Yang, E. Zhang, X. Li, Y. Zhang, J. Qu and Z.-Z. Yu, Carbon, 2016, 98, 50-57.

38 H. K. F. Cheng, N. G. Sahoo, Y. P. Tan, Y. Pan, H. Bao, L. Li, S. H. Chan and J. Zhao, ACS Appl. Mater. Interfaces, 2012, 4, 2387-2394.

39 H. Feng, X. Wang and D. Wu, Ind. Eng. Chem. Res., 2013, 52, 10160-10171.

40 S. Y. Kim, Y. J. Noh and J. Yu, Composites, Part A, 2015, 69, 219-225.

41 C.-W. Nan, G. Liu, Y. Lin and M. Li, Appl. Phys. Lett., 2004, 85, 3549-3551. 